Research Paper

\title{
Impact of the Age-adjusted Charlson comorbidity index on the short- and long-term outcomes of patients undergoing curative gastrectomy for gastric cancer
}

\author{
Yukio Maezawa ${ }^{1,2^{*}}$, Toru Aoyama ${ }^{1 *}$, Kazuki Kano ${ }^{1}$, Hiroshi Tamagawa ${ }^{1}$, Masakatsu Numata ${ }^{1}$, Kentaro \\ Hara $^{1}$, Masaaki Murakawa1,2, Takanobu Yamada1, 2, Tsutomu Sato ${ }^{1}$, Takashi Ogata ${ }^{2}$, Takashi Oshima ${ }^{1,2}$, \\ Norio Yukawa1 ${ }^{1}$, Takaki Yoshikawa1,2,3, Munetaka Masuda ${ }^{1}$, Yasushi Rino ${ }^{1 凶}$ \\ 1. Department of Surgery, Yokohama City University, 3-9, Fukuura, Kanazawa-ku, Yokohama, Kanagawa 236-0004, Japan \\ 2. Department of Gastrointestinal Surgery, Kanagawa Cancer Center, 2-3-2, Nakao, Asahi-ku, Yokohama, Kanagawa 241-8515, Japan. \\ 3. Department of Gastric Surgery, National Cancer Center Hospital, 5-1-1 Tsukiji, Chuo-Ku, Tokyo 104-0045, Japan. \\ *These two authors contributed equally. \\ $\triangle$ Corresponding author: Toru Aoyama, Department of Surgery, Yokohama City University, 3-9 Fukuura, Kanazawa-ku, Yokohama 236-0004, Japan. E-mail: \\ t-aoyama@lilac.plla.or.jp; Tel: 045-787-2800; Fax: 045-787-0226 \\ (C) The author(s). This is an open access article distributed under the terms of the Creative Commons Attribution License (https://creativecommons.org/licenses/by/4.0/). \\ See http://ivyspring.com/terms for full terms and conditions.
}

Received: 2019.04.03; Accepted: 2019.08.06; Published: 2019.08.29

\begin{abstract}
Background: The aim of this study was to determine whether or not the short- and long-term outcomes were affected by the age-adjusted Charlson comorbidity index (ACCl) in patients who underwent curative resection for gastric cancer.

Methods: The patients were retrospectively selected from among the medical records of consecutive patients who underwent curative gastrectomy with nodal dissection for gastric cancer at Yokohama City University and Kanagawa Cancer Center from January 2000 to August 2015.

Results: A total of 2254 patients were eligible for inclusion in the present study. One thousand six hundred fifty-six patients had an $\mathrm{ACCl}$ of $<6$ points (ACCI low group), while 598 had a score of $\geq 6$ points (ACCl high group). The median age $(\mathrm{p}<0.001)$ and American Society of Anesthesiologists physical status (ASA-PS) score $(p<0.001)$ of the $A C C l$ high group were higher in comparison to the $A C C l$ low group. The incidence of surgical complications in the $\mathrm{ACCl}$ high group was significantly higher than that in the $\mathrm{ACCl}$ low group $(12.0 \%$ vs. $7.2 \%, \mathrm{p}<0.001)$. Univariate and multivariate analyses demonstrated that an $\mathrm{ACCl}$ high classification was a significant risk factor for postoperative complications. In addition, the 5 -year $\mathrm{OS}$ rates of the $\mathrm{ACCl}$ low and $\mathrm{ACCl}$ high groups were $85.4 \%$ and $74.1 \%$, respectively. The difference was statistically significant $(p<0.001)$. The univariate and multivariate analyses demonstrated that an $\mathrm{ACCl}$ high classification was a significant prognostic factor for OS.

Conclusions: Our results support that a high $\mathrm{ACCl}$ value is an independent risk factor for the short- and long-term outcomes of patients with gastric cancer. To improve the survival of patients with gastric cancer, it is necessary to carefully plan the perioperative care and the surgical strategy according to the $\mathrm{ACCl}$.
\end{abstract}

Key words: Age-adjusted Charlson comorbidity index, comorbidity, postoperative complications, overall survival, gastric cancer

\section{Background}

A total of 951,600 new cases and 723,100 deaths due to gastric cancer occurred worldwide in 2012 [1]. Complete resection is essential for the cure of localized gastric cancer. However, the morbidity and

mortality rates of patients who undergo radical gastrectomy for gastric cancer are reported to be $20-40 \%$ and $1-5 \%$, respectively [2,3]. Recently, the proportion of elderly patients diagnosed with gastric 
cancer has tended to increase [4]. Elderly patients are likely to have more comorbidities and the presence of comorbidities is considered to be associated with a higher risk of morbidity and mortality [5]. Several previous studies have shown that the presence of comorbidities and their severity affects both the shortand long-term outcomes after gastric cancer surgery [6]. However, the optimal tools for comprehensively evaluating the various comorbid diseases in gastric cancer treatment have not been sufficiently established.

The Charlson comorbidity index (CCI), which was first proposed by Charlson et al. in 1987, has been extensively used to evaluate the impact of comorbidity in a variety of cancers and non-cancer conditions [7]. The CCI is a prognostic taxonomy that is considered to be useful for prognostic prediction by weighing and scoring each comorbidity disease. It was initially developed to account for the influence of patients' adverse medical conditions on longitudinal studies. The age-adjusted Charlson comorbidity index (ACCI), corrects the final CCI score for the age of the patient. Although several studies evaluated the clinical impacts of the ACCI in the patients with various types of malignancies, most previous studies have used and evaluated the data with relatively small sample sizes of less than 200 from a single institution. Small sample sizes data have many limitations, such as unspecified indications of surgery, heterogeneous populations, heterogeneous treatments, and description bias of surgical morbidity. To overcome such limitations associated with small sample sizes data, we focused on cases that were enrolled in large individual patients' data collecting from multi institutes $[8,9,10]$.

The aim of this study was to determine whether or not the short- and long-term outcomes of the patients in a large database who underwent curative resection for gastric cancer were affected by the ACCI. This study had the ultimate goal of evaluating and confirming the actual impact of ACCI on the treatment outcomes of gastric cancer patients who received curative surgery.

\section{Patients and Methods}

\section{Patients}

The patients were retrospectively selected from among the medical records of consecutive patients who underwent gastrectomy with nodal dissection for gastric cancer at Yokohama City University and Kanagawa Cancer Center from January 2000 to August 2015, according to the following criteria: (1) histologically proven gastric adenocarcinoma, (2) D2 or $\mathrm{D} 1+$ gastrectomy with curative lymph node dissection as the first treatment, and (3) achieved complete (R0) resection.

\section{Surgical procedure and pathological findings}

In principle, D2 gastrectomy was selected for T2T4 disease, whereas D1+ was selected for T1 cancer according to the Japanese gastric cancer treatment guidelines ver. 3 [11]. The resected specimens were histopathologically examined and staged according to the Japanese classification of gastric carcinoma: 3rd English edition [12].

\section{Definition of postoperative complications}

Postoperative complications of grade 2-5 according to the Clavien-Dindo classification that occurred during hospitalization and/or within 30 days after surgery were retrospectively determined from the patient's records [13]. Grade 1 complications were not evaluated in order to exclude the possibility of a description bias in the patient's records.

\section{Adjuvant treatment}

All patients who underwent radical gastrectomy from January 2000 to August 2006 were followed up at an outpatient clinic and received surgery alone. In July 2006, the Adjuvant Chemotherapy Trial of S-1 for Gastric Cancer (ACTS-GC) demonstrated the efficacy of S-1 as adjuvant chemotherapy for Japanese patients undergoing D2 curative gastrectomy for locally-advanced gastric cancer (pathological stage II or III disease) [14]. Based on the ACTS-GC trial, S-1 adjuvant chemotherapy became the standard treatment for patients with stages II and III gastric cancer.

\section{Follow-up}

Patients were followed up at outpatient clinics. In principle, hematological tests and physical examinations were performed at least every three months for the first three years after surgery and then every six months until five years after surgery. The serum CEA and CA19-9 levels were checked at least every three months for five years. Patients underwent a computed tomography (CT) examination every six months during the first three years after surgery and then every twelve months until five years after surgery.

\section{Measurement of the age-adjusted CharIson co morbidity index $(\mathrm{ACCl})$}

We used the comorbidity index developed by Charlson et al. to quantify baseline comorbidities [8]. The weighted age and comorbidity values are shown in Table 1. Information on pre-existing comorbidities that were present before the gastrectomy were available from the medical records of Yokohama City 
University and Kanagawa Cancer Center. The index is a weighted measure that incorporates 19 different medical categories, each of which is weighted according to its potential impact on mortality. Conditions with a weight of one included: myocardial infarction, congestive heart failure, peripheral vascular disease, cerebrovascular disease, dementia, chronic pulmonary disease (COPD), connective tissue disease, ulcer disease, mild liver disease and diabetes mellitus without end-organ damage. Conditions with a weight of two included: hemiplegia, moderate or severe chronic kidney disease, diabetes with end-organ damage, solid tumor, leukemia and lymphoma. Moderate or severe liver disease (e.g., cirrhosis with ascites) was given a weight of 3 and metastatic solid tumor or Acquired immunodeficiency syndrome (AIDS) was given a weight of 6 . The final score of each patient was calculated by taking all comorbid conditions into account. The ACCI was calculated with additional points added for age ( 1 point was added for each decade over 40 years of age). The basic value of " 2 " in the CCI was applied to all patients (because of gastric cancer). The patients were classified into the low or high ACCI groups according to their score: less than 6 (low ACCI), and 6 or more (high ACCI).

Table 1. Weighted index of comorbidities in Charlson comorbidity index

\begin{tabular}{ll}
\hline Comorbidities & Assigned weights for comorbidities \\
\hline Myocardial infarction & 1 \\
Congestive heart failure & 1 \\
Peripheral vascular disease & 1 \\
Cerebrovascular disease & 1 \\
Dementia & 1 \\
Chronic obstructive pulmonary disease & 1 \\
(COPD) & 1 \\
Connective tissue disease & 1 \\
Ulcer disease & 1 \\
Mild liver disease & 1 \\
Diabetes mellitus without end-organ damage & 2 \\
Hemiplegia & 2 \\
Moderate to severe chronic kidney disease & 2 \\
Diabetes with end-organ damage & 2 \\
Solid tumor & 2 \\
Leukemia & 2 \\
Lymphoma & 3 \\
Moderate to severe liver disease & 6 \\
Metastatic solid tumor & 6 \\
Acquired immunodeficiency syndrome & \\
(AIDS) &
\end{tabular}

Add 1 point per decade to ages over 40 years.

\section{Statistical analyses}

The significance of the correlation between the ACCI and clinicopathological parameters was determined using Fisher's exact test or the $\chi^{2}$ test and the Mann-Whitney $U$ test for continuous variables. Overall survival (OS) was defined as the period between surgery and death. Recurrence-free survival
(RFS) was defined as the period between surgery and recurrence or death, whichever came first. The data of the patients who did not experience an event was censored on the date of the final observation. The OS and RFS were evaluated by univariate and multivariate analyses, and the OS and RFS curves were calculated using the Kaplan-Meier method and compared by the log-rank test. Cox's proportional hazard model was used to perform univariate and multivariate survival analyses. To select a model, we used backward elimination in the multivariate analysis. The impact of the ACCI on postoperative morbidity and mortality were examined using Fisher's exact test or the $\chi^{2}$ test. Postoperative morbidity was evaluated by univariate and multivariate analyses and a logistic regression analysis was performed. Patients with missing covariate values were excluded. Standard clinical thresholds were used, dividing the continuous variables into no more than two categories. $P$ values of $<0.05$ were considered to indicate statistical significance. The survival data were obtained from hospital records or from the city registry system. The SPSS software program (ver. 23.0; IBM Corp., Armonk, NY, USA) was used to perform all of the statistical analyses. This study was approved by the Institutional Review Board (IRB) of the Yokohama City University (IRB Number: B160707003) and Kanagawa Cancer Center (2016.epidemiologic study-22).

\section{Results}

\section{The background characteristics of the patients}

A total of 2254 patients were eligible for inclusion in the present study. The patients' ages ranged from 24 to 87 years (median: 65 years); 1539 were male, and 715 were female. The American Society of Anesthesiologists physical status (ASA-PS) values of the patients were as follows ASA-PS 1, n= 860 (38.2\%); ASA-PS 2, $\mathrm{n}=1371$ (60.8\%); and ASA-PS 3, $\mathrm{n}=23(1.0 \%)$. The median follow-up period was 61 months (1-180 months). Sixty percent of the patients received distal gastrectomy, and $80 \%$ of patients received D2 lymph node dissection. One thousand six hundred fifty-six patients had an ACCI of $<6$ points and were classified into the ACCI low group; 598 had an ACCI of $\geq 6$ points and were classified into the ACCI high group. Table 2 summarizes the patients' demographic information and compares the clinical characteristics between the ACCI low and ACCI high groups. The median age $(p<0.001)$ and ASA-PS $(p<0.001)$ of the ACCI high group were higher than those in the ACCI low group. There were significant differences in operation time and blood loss between 
the two groups; however, both differences were small. There was no significant difference in the pathological stage. We also analyzed the relationship between the
ACCI value and every TNM stage ( $7^{\text {th }}$ edtion), and there was no relation between the ACCI value and every TNM stage $(p=0.089)[15]$.

Table 2. Comparison of patient background factors between patients with an age-adjusted Charlson comorbidity index value of $<6$ and those with an age-adjusted Charlson comorbidity Index value of $\geq 6$

\begin{tabular}{|c|c|c|c|c|}
\hline Variables & All patients $(n=2254)$ & ACCI<6 group $(n=1656)$ & $\mathrm{ACCI} \geq 6$ group $(\mathrm{n}=598)$ & $p$ value \\
\hline Age (years), median (range) & $65(24-87)$ & $62(24-79)$ & $74(60-87)$ & $<0.001$ \\
\hline Gender & & & & 0.008 \\
\hline Female & $715(31.7 \%)$ & $551(33.3 \%)$ & $164(27.4 \%)$ & \\
\hline Male & $1539(68.3 \%)$ & $1105(66.7 \%)$ & $434(72.6 \%)$ & \\
\hline Body mass index, median (range) & $22.3(13.7-39.5)$ & $22.2(13.7-39.5)$ & $22.7(14.8-33.0)$ & 0.001 \\
\hline ASA-PS & & & & $<0.001$ \\
\hline 1 & $860(38.2 \%)$ & $831(50.2 \%)$ & $29(4.8 \%)$ & \\
\hline 2 & $1371(60.8 \%)$ & $819(49.5 \%)$ & $552(92.3 \%)$ & \\
\hline 3 & $23(1.0 \%)$ & $6(0.4 \%)$ & $17(2.8 \%)$ & \\
\hline Surgical procedure & & & & 0.140 \\
\hline Total & $793(35.2 \%)$ & $568(34.3 \%)$ & $225(37.6 \%)$ & \\
\hline Distal & $1390(61.7 \%)$ & $1040(62.8 \%)$ & $350(58.5 \%)$ & \\
\hline Others & $71(3.1 \%)$ & $48(2.9 \%)$ & $23(3.8 \%)$ & \\
\hline Nodal dissection & & & & $<0.001$ \\
\hline $\mathrm{D} 1+$ & $482(21.4 \%)$ & $315(19.0 \%)$ & $167(27.9 \%)$ & \\
\hline D2 & $1772(78.6 \%)$ & $1341(81.0 \%)$ & $431(71.1 \%)$ & \\
\hline Operation time (min), median (range) & $190(68-731)$ & $190(68-731)$ & 195 (71-609) & 0.005 \\
\hline Blood loss (ml), median (range) & $135(5-2510)$ & $130(5-2510)$ & $150(5-2230)$ & 0.006 \\
\hline Pathological Stage & & & & 0.540 \\
\hline $\mathrm{I}$ & $1440(63.9 \%)$ & $1067(64.4 \%)$ & $373(62.4 \%)$ & \\
\hline II & $395(17.5 \%)$ & $290(17.5 \%)$ & $105(17.6 \%)$ & \\
\hline III & $419(18.6 \%)$ & $299(18.1 \%)$ & $120(20.1 \%)$ & \\
\hline Postoperative surgical complication & & & & 0.009 \\
\hline No & $2079(92.2 \%)$ & $1542(93.1 \%)$ & $537(89.8 \%)$ & \\
\hline Yes & $175(7.8 \%)$ & $114(6.9 \%)$ & $61(10.2 \%)$ & \\
\hline Adjuvant chemohetrapy & & & & 0.750 \\
\hline No & $1823(80.9 \%)$ & $1342(81.0 \%)$ & $481(80.4 \%)$ & \\
\hline Yes & $431(19.1 \%)$ & $314(19.0 \%)$ & $117(19.6 \%)$ & \\
\hline Absolute CCI values, median (range) & $2(2-4)$ & $2(2-4)$ & $3(2-4)$ & $<0.001$ \\
\hline pT stages & & & & 0.001 \\
\hline 1 & $1313(58.3 \%)$ & $975(58.8 \%)$ & $338(56.5 \%)$ & \\
\hline 2 & $318(14.1 \%)$ & $228(13.8 \%)$ & $90(15.1 \%)$ & \\
\hline 3 & $197(8.7 \%)$ & $133(8.0 \%)$ & $64(10.7 \%)$ & \\
\hline 4 & $426(18.9 \%)$ & $320(19.4 \%)$ & $106(17.7 \%)$ & \\
\hline pN stages & & & & 0.306 \\
\hline 0 & $1530(67.9 \%)$ & $1113(68.4 \%)$ & $397(66.4 \%)$ & \\
\hline 1 & $279(12.4 \%)$ & $202(12.2 \%)$ & $77(12.9 \%)$ & \\
\hline 2 & $204(9.1 \%)$ & $138(8.3 \%)$ & $66(11.0 \%)$ & \\
\hline 3 & $241(10.7 \%)$ & $183(11.1 \%)$ & $58(2.5 \%)$ & \\
\hline Number of dissected lymph nodes, median (range) & $45(0-184)$ & $46(0-184)$ & $42(3-139)$ & 0.001 \\
\hline Frequency of lymphatic vessel infiltration & & & & 0.090 \\
\hline No & $1606(71.3 \%)$ & $1196(72.2 \%)$ & $410(68.6 \%)$ & \\
\hline Yes & $648(28.7 \%)$ & $460(27.8 \%)$ & $188(31.4 \%)$ & \\
\hline Lauren histological type & & & & $<0.001$ \\
\hline Intestinal & $1032(45.8 \%)$ & $704(42.5 \%)$ & $328(54.8 \%)$ & \\
\hline Diffuse & $1222(54.2 \%)$ & $952(57.5 \%)$ & $270(45.2 \%)$ & \\
\hline Tumor size (mm), median (range) & $37(0-212)$ & $35.5(0-212)$ & $38(0-210)$ & 0.142 \\
\hline Number of minimal invasive procedures & & & & 0.621 \\
\hline Open & $1683(74.7 \%)$ & $1241(74.9 \%)$ & $442(73.9 \%)$ & \\
\hline Laparoscopic & $571(25.3 \%)$ & $415(25.1 \%)$ & $156(26.1 \%)$ & \\
\hline Length of hospital stay (in days), median (range) & $10(2-172)$ & $10(6-137)$ & $10(2-172)$ & 0.844 \\
\hline Surgical extension to other organs & & & & 0.610 \\
\hline No & $2227(98.8 \%)$ & $1635(98.7 \%)$ & $592(99.0 \%)$ & \\
\hline Yes & $27(1.2 \%)$ & $21(1.3 \%)$ & $6(1.0 \%)$ & \\
\hline Frequency of redo surgery & & & & 0.136 \\
\hline No & $2210(98.0 \%)$ & $1628(98.3 \%)$ & $582(97.3 \%)$ & \\
\hline Yes & $44(2.0 \%)$ & $28(1.7 \%)$ & $16(2.7 \%)$ & \\
\hline Distribution of the respective Clavien Dindo stages & & & & $<0.001$ \\
\hline $0-\mathrm{I}$ & $1957(86.8 \%)$ & $1470(88.8 \%)$ & $487(81.4 \%)$ & \\
\hline II & $194(8.6 \%)$ & $122(7.4 \%)$ & $72(12.0 \%)$ & \\
\hline IIIa & $55(2.4 \%)$ & $36(2.2 \%)$ & $19(3.2 \%)$ & \\
\hline $\mathrm{IIIlb}$ & $35(1.5 \%)$ & $22(1.3 \%)$ & $13(2.2 \%)$ & \\
\hline $\mathrm{IVa}$ & $8(0.4 \%)$ & $5(0.3 \%)$ & $3(0.5 \%)$ & \\
\hline $\mathrm{IVb}$ & $1(0.04 \%)$ & $1(0.1 \%)$ & $0(0.0 \%)$ & \\
\hline
\end{tabular}




\begin{tabular}{|c|c|c|c|c|c|c|c|}
\hline $\mathrm{V}$ & & & & & & $4(0.2 \%)$ & \\
\hline $\begin{array}{l}\text { ASA-PS America } \\
\text { Table } \mathbf{3} \text {. Un } \\
\text { of the clinice } \\
\text { complications }\end{array}$ & $\begin{array}{l}\text { Society of } \\
\text { ariate an } \\
\text { athologi }\end{array}$ & $\begin{array}{l}\text { Anesth } \\
\text { d mi } \\
\text { cal fa }\end{array}$ & $\begin{array}{l}\text { esiologists } \\
\text { altivariat } \\
\text { ctors as }\end{array}$ & $\begin{array}{l}\text { physical } \\
\text { logisti } \\
\text { sociate }\end{array}$ & $\begin{array}{l}\text { status, } \\
\text { creg } \\
\text { wit }\end{array}$ & $\begin{array}{l}\text { CCI Charl } \\
\text { ression } \\
\text { h posto }\end{array}$ & $\begin{array}{l}\text { on comork } \\
\text { inalyses } \\
\text { erative }\end{array}$ \\
\hline Characteristics & Number & Univ & ariate & & Mult & tivariate & \\
\hline & & $\overline{\mathrm{HR}}$ & $95 \% \mathrm{CI}$ & P value & HR & $95 \% \mathrm{CI}$ & P value \\
\hline Age (years) & & & & 0.016 & & & \\
\hline$<65$ & 1038 & 1.00 & & & & & \\
\hline$\geq 65$ & 1216 & 1.46 & $1.07-1.98$ & & & & \\
\hline Gender & & & & $<0.001$ & & & 0.002 \\
\hline Female & 715 & 1.00 & & & 1.00 & & \\
\hline Male & 1539 & 2.04 & $1.41-2.95$ & & 1.84 & $1.25-2.71$ & \\
\hline Body mass index & & & & 0.007 & & & \\
\hline$<25$ & 1831 & 1.00 & & & & & \\
\hline$\geq 25$ & 423 & 1.61 & $1.14-2.26$ & & & & \\
\hline Operation time & & & & $<0.001$ & & & $<0.001$ \\
\hline$<190$ min & 1131 & 1.00 & & & 1.00 & & \\
\hline$\geq 190 \mathrm{~min}$ & 1123 & 2.13 & $1.56-2.91$ & & 2.00 & $1.45-2.76$ & \\
\hline Blood loss & & & & 0.017 & & & 0.003 \\
\hline$<140 \mathrm{ml}$ & 1131 & 1.00 & & & 1.00 & & \\
\hline$\geq 140 \mathrm{ml}$ & 1123 & 1.92 & $1.41-2.62$ & & 1.62 & $1.17-2.22$ & \\
\hline Nodal dissection & & & & 0.44 & & & \\
\hline D2 & 1772 & 1.00 & & & & & \\
\hline D1+ & 482 & 1.15 & $0.81-1.63$ & & & & \\
\hline ASA-PS & & & & 0.002 & & & \\
\hline 1 & 860 & 1.00 & & & & & \\
\hline $2-3$ & 1394 & 1.67 & $1.20-2.32$ & & & & \\
\hline ACCI & & & & $<0.001$ & & & 0.002 \\
\hline$<6$ & 1656 & 1.00 & & & 1.00 & & \\
\hline$\geq 6$ & 598 & 1.77 & $1.30-2.41$ & & 1.66 & $1.21-2.28$ & \\
\hline
\end{tabular}

ASA-PS American Society of Anesthesiologists physical status, ACCI Age-adjusted Charlson comorbidity index

Table 4. Univariate and multivariate Cox proportional hazards analyses of the clinicopathological factors associated with overall survival

\begin{tabular}{|c|c|c|c|c|c|c|c|}
\hline \multirow[t]{2}{*}{ Characteristics } & \multirow[t]{2}{*}{ Number } & \multicolumn{3}{|c|}{ Univariate } & \multicolumn{3}{|c|}{ Multivariate } \\
\hline & & $\overline{\mathrm{HR}}$ & $95 \% \mathrm{CI}$ & P value & HR & $95 \% \mathrm{CI}$ & $P$ value \\
\hline \multicolumn{4}{|l|}{ Age(years) } & $<0.001$ & & & \\
\hline$<65$ & 1038 & 1.00 & & & & & \\
\hline$\geq 65$ & 1216 & 1.47 & $1.21-1.80$ & & & & \\
\hline \multicolumn{2}{|l|}{ Gender } & & & 0.048 & & & 0.020 \\
\hline Female & 715 & 1.00 & & & 1.00 & & \\
\hline Male & 1539 & 1.25 & $1.00-1.56$ & & 1.32 & $1.04-1.66$ & \\
\hline \multicolumn{2}{|c|}{ Pathological stage } & & & $<0.001$ & & & $<0.001$ \\
\hline I & 1440 & 1.00 & & & 1.00 & & \\
\hline II & 395 & 3.07 & $2.34-4.03$ & & 2.84 & $2.16-3.73$ & \\
\hline III & 419 & 7.55 & $6.00-9.51$ & & 6.02 & $4.73-7.66$ & \\
\hline \multicolumn{2}{|c|}{ Infectious complication } & & & 0.015 & & & 0.012 \\
\hline No & 2079 & 1.00 & & & 1.00 & & \\
\hline Yes & 175 & 1.50 & $1.08-2.07$ & & 1.52 & $1.10-2.11$ & \\
\hline \multicolumn{2}{|l|}{ ASA-PS } & & & $<0.001$ & & & \\
\hline 1 & 860 & 1.00 & & & & & \\
\hline $2-3$ & 1394 & 1.51 & $1.23-1.86$ & & & & \\
\hline \multicolumn{2}{|l|}{ ACCI§ } & & & $<0.001$ & & & $<0.001$ \\
\hline$<6$ & 1656 & 1.00 & & & 1.00 & & \\
\hline$\geq 6$ & 598 & 1.73 & $1.40-2.13$ & & 1.80 & $1.46-2.23$ & \\
\hline
\end{tabular}

\section{Postoperative complications}

Postoperative complications were found in 191 $(8.5 \%)$ of the 2254 patients. The most frequent postoperative complication was pancreatic fistula $(2.8 \%)$, followed by anastomotic leakage $(1.8 \%)$, anatomic stenosis $(1.7 \%)$, ileus $(1.3 \%)$, and surgical site infection (SSI) $(1.1 \%)$. These complications were
$0(0.0 \%) \quad 4(0.7 \%)$

index, ACCI Age-adjusted Charlson comorbidity index

more frequent than $1.0 \%$. Surgical mortality was observed in four patients, all of whom were in the ACCI high group. The incidence of surgical complications in the ACCI high group was significantly higher than that in the ACCI low group $(12.0 \%$ vs. $7.2 \%, \mathrm{p}<0.001)$. Infectious complications, such as pancreatic fistula, anastomotic leakage, SSI, pneumonia, abdominal abscess and catheter infection, were significantly more frequent in the ACCI high group $(p=0.009)$. Interestingly, the incidence of pancreatic fistula in the ACCI high group was significantly higher than that in the ACCI low group. However, the incidence of pancreatic fistula was not related to somehow more extensive surgical procedure such as the extent of nodal dissection $(p=0.47)$, surgical extension to pancreatic tail $(p=0.78)$ and frequency of far advanced tumors (T4a/T4b or not; $p=0.53$ ). On the other hand, the incidence of non-infectious complications, such as anatomic stenosis, ileus and lymphatic fistula, did not differ between the two groups to a statistically significant extent $(p=0.22)$. The univariate and multivariate analyses demonstrated that an ACCI value of $\geq 6$ was a significant risk factor for postoperative complications (HR 1.66; $95 \%$ CI 1.21-2.28, P = 0.002) (Table 4). The median was used for the boundaries of operation time and blood loss.

\section{Survival analyses}

The median follow-up time for the 2254 patients was 60 (range: 0-177) months. The 5-year OS rates of the ACCI low and ACCI high groups were $85.4 \%$ and $74.1 \%$, respectively. The difference was statistically significant $(p<0.001)$. The OS curves are shown in Figure 1. Two hundred thirty-eight of 406 patients died of gastric cancer within this period. The OS was significantly worse in the ACCI high group, with an unadjusted hazard ratio (HR) for complications of 1.73 (95 \%confidence interval [CI] 1.40-2.13; $\mathrm{P}<0.0001)$. Before multivariate analysis, $\mathrm{T}$ and $\mathrm{N}$ factor (instead of pStage) were excluded to alleviate the multicollinearity. The univariate and multivariate analyses demonstrated that an ACCI value of $\geq 6$ was a significant prognostic factor for OS (HR 1.80; $95 \%$ CI 1.46-2.23, $\mathrm{P}<0.001)$. The ASA-PS was not a significant prognostic factor (Table 4).

The 5-year RFS rates in the ACCI low and ACCI high groups were $83.1 \%$ and $73.4 \%$, respectively. The difference was statistically significant $(p<0.001)$. The RFS curves are shown in Figure 2. The univariate and multivariate analyses also demonstrated an ACCI value of $\geq 6$ was a significant prognostic factor for RFS (HR 1.44; $95 \%$ CI 1.15-1.80, P = 0.001). The 5-year 
cancer specific overall survival (CSS) in the ACCI low

respectively. The difference was statistically and ACCI high groups were $89.1 \%$ and $85.7 \%$, significant $(\mathrm{p}=0.016)$.

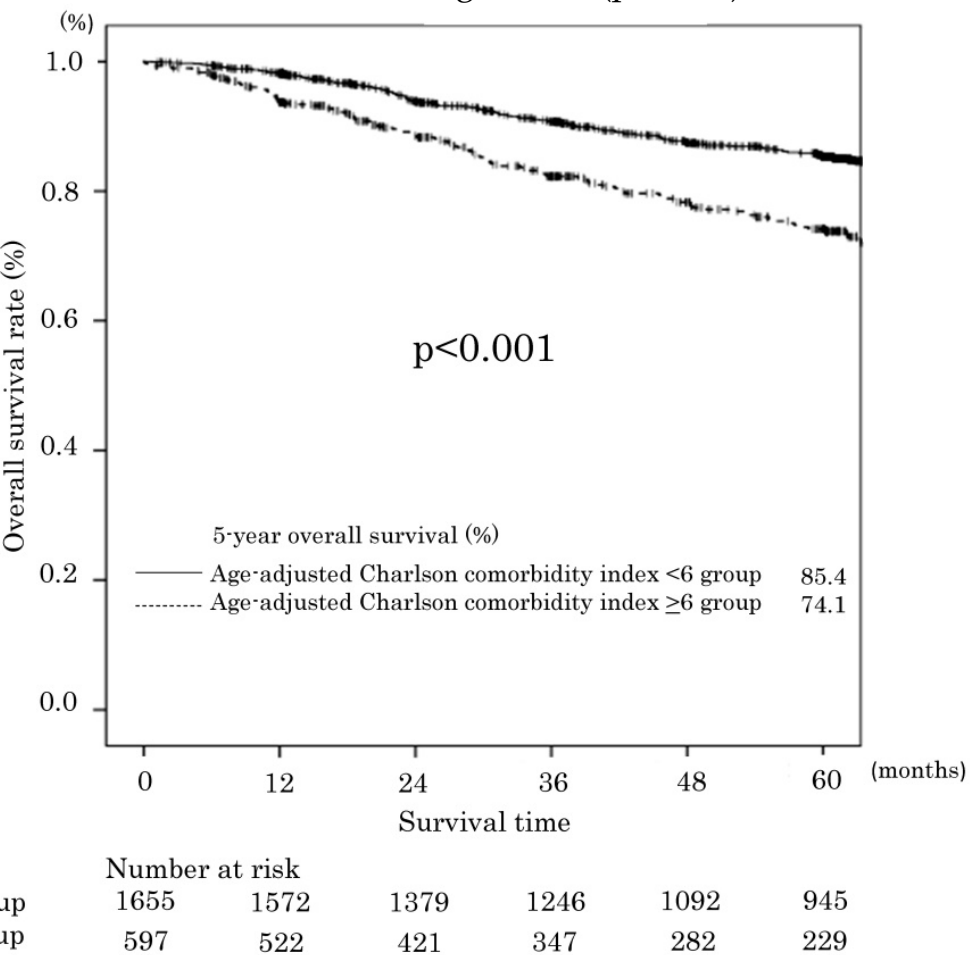

Figure 1. Overall survival in the age-adjusted Charlson comorbidity index $<6$ and $\geq 6$ groups.

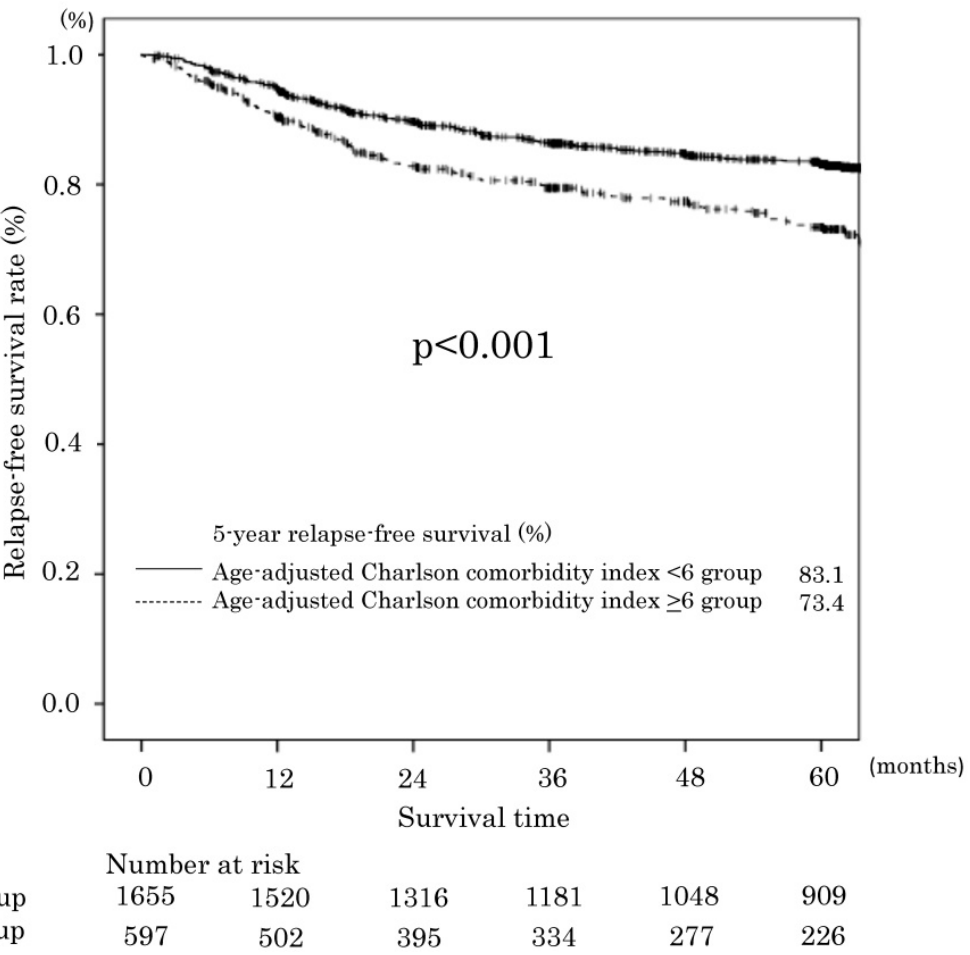

Figure 2. Relapse-free survival in the age-adjusted Charlson_comorbidity index $<6$ and $\geq 6$ groups.

\section{Discussion}

The present study aimed to determine whether or not the ACCI had a clinical impact on gastric cancer patients who underwent curative gastrectomy. The major finding of the present study was that both the short- and long-term outcomes of gastric cancer patients were affected by the ACCI. Our results support that a high ACCI was an independent risk factor for postoperative complications and poorer OS. 
To improve the survival of gastric cancer patients, it is necessary to carefully plan the surgical procedure, perioperative care and the surgical strategy using the ACCI.

The present study demonstrated that the ACCI was an independent risk factor for surgical complications in patients who underwent curative surgery for gastric cancer. The hazard ratio for surgical complications was 1.66 (95\% confidence interval, 1.21 to 2.28). Similar results have been observed in other malignancies. Kahl et al. analyzed the prognostic impact of the ACCI on both postoperative morbidity and overall survival (OS) in 793 patients with advanced epithelial ovarian cancer [16]. The patients were classified into low ACCI (0-1), intermediate ACCI (2-3), and high ACCI (>4) groups. They demonstrated that intermediate and high ACCI values were significantly associated with severe postoperative complications, defined as Clavien-Dindo classification grade 3-5 (Intermediate ACCI: OR 1.55, 95\% CI 1.06-2.26, p=0.026, High ACCI: OR 3.27, 95\% CI 1.97-5.43, p<0.001). Other reports showed similar results [9] [17]. In addition, the incidence of infectious complications in the ACCI high group was significantly higher than that in the ACCI low group $(p=0.001)$, while the incidence of noninfectious complications did not differ to a statistically significant extent. Thus, careful attention should be paid to the possible development of surgical complications, especially infectious complications, in patients with high ACCI values when surgeons perform curative gastrectomy for gastric cancer.

The present study also demonstrated that the ACCI was an independent risk factor for OS in the patients who underwent curative surgery for gastric cancer. There are several possible reasons why the ACCI might have affected the long-term outcomes of gastric cancer patients. One possible reason is that the ACCI might be associated with postoperative surgical complications. As mentioned above, the incidence of postoperative infectious complications in the ACCI high group was higher than that in the ACCI low group in the present study. Recent studies have demonstrated that the development of postoperative complications is associated with decreased survival or an increased risk of disease recurrence in various types of malignancies [18-26]. Actually, we previously investigated the impact of postoperative complications on gastric cancer survival and recurrence after curative surgery [27]. Another possible reason for this association is that the patients who were in the ACCI high group might have had some factors that led to decreased host immunity against their tumors. For example, Goldfarb et al. reported that treatment aimed at the perioperative enhancement of cell-mediated immunity with the simultaneous inhibition of excessive catecholamine and prostaglandin responses could be successful in limiting postoperative immune suppression and metastatic progression [28]. In addition, Dunn et al. suggested that the adaptive immune system could function by identifying and eliminating nascent tumor cells in experimental models [29]. However, these mechanisms were speculative, and further work is clearly needed to investigate them.

Present study also demonstrated the relation of ACCI and postoperative complications rate. Pancreatic fistula occurred in the most patients and the incidence was $2.8 \%$ in all patients. Furthermore, present study demonstrated the significantly high incidence of pancreatic fistula in the ACCI high group $(4.3 \%)$ than that in the ACCI low group $(2.3 \%)$ was shown and the higher pancreatic fistula rate in the ACCI high group was indeed related to comorbidity and not to a somehow more extensive surgical procedure. However, we used the fistula grading according to Clavien- Dindo (not the Bassi classification). Therefore, the frequency of pancreatic fistula might be underestimated.

Although the present results are considered to be solid in terms of the follow-up period and sample size, the present study is associated with several limitations. First, the present study was retrospective in nature, and might have contained a selection bias. Second, there was a time bias in this study, as the data were collected over a relatively long period (20002015), the surgical procedures, perioperative care, and adjuvant chemotherapy might have changed over the years [30] [11]. Third, the definition and severity of morbidities were not strictly defined in this study. The postoperative surgical complications were reported based on the judgment of individual physicians rather than the study protocol. Although the incidence of morbidity in this study was similar to that in other large studies, the incidence of some surgical complications might have been underestimated [31, 32]. A further important limitation of all of the available data regarding ACCI, including the date used in the current study, is the lack of consensus regarding the most appropriate cut-off for the evaluation of the ACCI [10, 16] [33] [34]. In our study, we used a cut-off value of 6 according to previous reports that evaluated all endpoints. However, there needs to be a consensus regarding the definitions of 'high' versus 'low' ACCI. This would also greatly aid the use of the ACCI as a stratification factor in future studies. 


\section{Conclusion}

The short- and long-term outcomes of gastric cancer patients were affected by the ACCI. Our results support that a high ACCI was an independent risk factor for postoperative complications and poorer OS. To improve the survival of gastric cancer patients, it is necessary to carefully plan the perioperative care and the surgical strategies using the ACCI in daily clinical practice.

\section{Abbreviations}

ACCI: age-adjusted Charlson comorbidity index; ACTS-GC: Adjuvant Chemotherapy Trial of S-1 for Gastric Cancer; AIDS: Acquired immunodeficiency syndrome; ASA-PS: American Society of Anesthesiologists physical status; CA19-9: carbohydrate antigen 19-9; CCI: Charlson comorbidity index; CEA: Carcinoembryonic antigen; CI: Confidence interval; COPD: Chronic obstructive pulmonary disease; CT: computed tomography; OR: Odds ratio; OS: Overall survival; RFS: Recurrence-free survival; SSI: surgical site infection.

\section{Acknowledgments}

The authors express their sincere gratitude to Ms. Minako Igarashi, Ms. Natsumi Sato and Ms. Rika Takahashi for their excellent data management in this study.

\section{Availability of data and materials}

The datasets analyzed during the current study are available from the corresponding author on reasonable request.

\section{Authors' contributions}

YM, TA and YR made substantial contributions to conception and design. $\mathrm{YM}, \mathrm{TA}, \mathrm{KK}, \mathrm{MN}, \mathrm{SS}, \mathrm{SA}$, JM, TH, TY, TS, TO, NY, TY, and YR made substantial contributions to acquisition of data, or analysis and interpretation of data. YM, TA, TH, TY, NY, TY and YR have been involved in drafting the manuscript or revising it critically for important intellectual content. TA, YR, and MM have given final approval of the version to be published. Each author should have participated sufficiently in the work to take public responsibility for appropriate portions of the content; and agreed to be accountable for all aspects of the work in ensuring that questions related to the accuracy or integrity of any part of the work are appropriately investigated and resolved. All authors read and approved the final manuscript.

\section{Ethics approval and consent to participate}

This retrospective study was approved by the Institutional Review Board (IRB) of the Yokohama
City University (IRB Number: B160707003) and Kanagawa Cancer Center (2016.epidemiologic study-22). Based on this IRB-approval, we retrospectively collected clinical data of patients who received surgery during Jan 2000 and Aug 2015. Because the study was retrospective study without any investigational intervention, the study-specific informed consent was not obtained from each patient. Instead, we obtained the comprehensive written informed consent for retrospective study from all patients since 2010, including the patients who entered into the present study. This policy was in compliance with Helsinki Declaration of 1975, as revised in 1983 and with Japanese ethical guideline for clinical studies 2014. Recently, revised Japanese ethical guideline for clinical studies was applied since June 2017 and informed consent was principally mandatory as long as no difficulty for acquisition of consent even for retrospective study, however, the data collection of the present study had been finished until Jan 2016.

\section{Competing Interests}

The authors have declared that no competing interest exists.

\section{References}

1. Torre LA, Bray F, Siegel RL, Ferlay J, Lortet-Tieulent J, Jemal A: Global cancer statistics, 2012. CA Cancer J Clin 2015, 65:87-108.

2. Yoshikawa T, Rino Y, Yukawa N, Oshima T, Tsuburaya A, Masuda M: Neoadjuvant chemotherapy for gastric cancer in Japan: a standing position by comparing with adjuvant chemotherapy. Surg Today 2014, 44:11-21.

3. Aoyama T, Yoshikawa T: Adjuvant therapy for locally advanced gastric cancer. Surg Today 2017.

4. Kitamura K, Yamaguchi T, Taniguchi H, Hagiwara A, Yamane T, Sawai K, Takahashi T: Clinicopathological characteristics of gastric cancer in the elderly. Br J Cancer 1996, 73:798-802.

5. Hamakawa T, Kurokawa Y, Mikami J, Miyazaki Y, Takahashi T, Yamasaki M, Miyata H, Nakajima K, Takiguchi S, Mori M, Doki Y: Risk factors for postoperative complications after gastrectomy in gastric cancer patients with comorbidities. Surg Today 2016, 46:224-228.

6. Inokuchi M, Kato K, Sugita H, Otsuki S, Kojima K: Impact of comorbidities on postoperative complications in patients undergoing laparoscopy-assisted gastrectomy for gastric cancer. BMC Surg 2014, 14:97.

7. Charlson ME, Pompei P, Ales KL, MacKenzie CR: A new method of classifying prognostic comorbidity in longitudinal studies: development and validation. J Chronic Dis 1987, 40:373-383.

8. Charlson M, Szatrowski TP, Peterson J, Gold J: Validation of a combined comorbidity index. J Clin Epidemiol 1994, 47:1245-1251.

9. Dias-Santos D, Ferrone CR, Zheng H, Lillemoe KD, Fernandez-Del Castillo C: The Charlson age comorbidity index predicts early mortality after surgery for pancreatic cancer. Surgery 2015, 157:881-887.

10. Tian Y, Jian Z, Xu B, Liu H: Age-adjusted Charlson comorbidity index score as predictor of survival of patients with digestive system cancer who have undergone surgical resection. Oncotarget 2017, 8:79453-79461.

11. Japanese gastric cancer treatment guidelines 2010 (ver. 3). Gastric Cancer 2011, 14:113-123.

12. Japanese classification of gastric carcinoma: 3rd English edition. Gastric Cancer 2011, 14:101-112.

13. Dindo D, Demartines $\mathrm{N}$, Clavien PA: Classification of surgical complications: a new proposal with evaluation in a cohort of 6336 patients and results of a survey. Ann Surg 2004, 240:205-213.

14. Sakuramoto S, Sasako M, Yamaguchi T, Kinoshita T, Fujii M, Nashimoto A, Furukawa H, Nakajima T, Ohashi Y, Imamura H, et al: Adjuvant chemotherapy for gastric cancer with S-1, an oral fluoropyrimidine. $N$ Engl $J$ Med 2007, 357:1810-1820.

15. Edge SB BD, Compton CC, Fritz AG, Greene FL, Trotti A, editors: AJCC Cancer Staging Manual. Seventh Edition. New York, NY. Springer 2010.

16. Kahl A, du Bois A, Harter P, Prader S, Schneider S, Heitz F, Traut A, Alesina PF, Meier B, Walz M, et al: Prognostic Value of the Age-Adjusted Charlson 
Comorbidity Index (ACCI) on Short- and Long-Term Outcome in Patients with Advanced Primary Epithelial Ovarian Cancer. Ann Surg Oncol 2017, 24:3692-3699.

17. Tian $\mathrm{Y}, \mathrm{Xu}$ B, Yu G, Li Y, Liu H: Age-adjusted charlson comorbidity index score as predictor of prolonged postoperative ileus in patients with colorectal cancer who underwent surgical resection. Oncotarget 2017, 8:20794-20801.

18. Lagarde SM, de Boer JD, ten Kate FJ, Busch OR, Obertop H, van Lanschot JJ: Postoperative complications after esophagectomy for adenocarcinoma of the esophagus are related to timing of death due to recurrence. Ann Surg 2008, 247:71-76.

19. Lerut T, Moons J, Coosemans W, Van Raemdonck D, De Leyn P, Decaluwe H, Decker G, Nafteux P: Postoperative complications after transthoracic esophagectomy for cancer of the esophagus and gastroesophageal junction are correlated with early cancer recurrence: role of systematic grading of complications using the modified Clavien classification. Ann Surg 2009, 250:798-807.

20. McArdle CS, McMillan DC, Hole DJ: Impact of anastomotic leakage on long-term survival of patients undergoing curative resection for colorectal cancer. Br J Surg 2005, 92:1150-1154.

21. Mirnezami A, Mirnezami R, Chandrakumaran K, Sasapu K, Sagar P, Finan P: Increased local recurrence and reduced survival from colorectal cancer following anastomotic leak: systematic review and meta-analysis. Ann Surg 2011, 253:890-899.

22. Mynster T, Christensen IJ, Moesgaard F, Nielsen HJ: Effects of the combination of blood transfusion and postoperative infectious complications on prognosis after surgery for colorectal cancer. Danish RANX05 Colorectal Cancer Study Group. Br J Surg 2000, 87:1553-1562.

23. Walker KG, Bell SW, Rickard MJ, Mehanna D, Dent OF, Chapuis PH, Bokey EL: Anastomotic leakage is predictive of diminished survival after potentially curative resection for colorectal cancer. Ann Surg 2004, 240:255-259.

24. Sierzega M, Kolodziejczyk P, Kulig J: Impact of anastomotic leakage on long-term survival after total gastrectomy for carcinoma of the stomach. $\mathrm{BrJ}$ Surg 2010, 97:1035-1042.

25. Tokunaga M, Tanizawa $\mathrm{Y}$, Bando E, Kawamura T, Terashima M: Poor survival rate in patients with postoperative intra-abdominal infectious complications following curative gastrectomy for gastric cancer. Ann Surg Oncol 2013, 20:1575-1583.

26. Tsujimoto H, Ichikura T, Ono S, Sugasawa H, Hiraki S, Sakamoto N, Yaguchi Y, Yoshida K, Matsumoto Y, Hase K: Impact of postoperative infection on long-term survival after potentially curative resection for gastric cancer. Ann Surg Oncol 2009, 16:311-318.

27. Hayashi T, Yoshikawa T, Aoyama T, Hasegawa S, Yamada T, Tsuchida K, Fujikawa H, Sato T, Ogata T, Cho H, et al: Impact of infectious complications on gastric cancer recurrence. Gastric Cancer 2015, 18:368-374.

28. Goldfarb Y, Sorski L, Benish M, Levi B, Melamed R, Ben-Eliyahu S: Improving postoperative immune status and resistance to cancer metastasis: a combined perioperative approach of immunostimulation and prevention of excessive surgical stress responses. Ann Surg 2011, 253:798-810.

29. Dunn GP, Old LJ, Schreiber RD: The immunobiology of cancer immunosurveillance and immunoediting. Immunity 2004, 21:137-148.

30. Nakajima T: Gastric cancer treatment guidelines in Japan. Gastric Cancer 2002, 5:1-5.

31. Kim HH, Hyung WJ, Cho GS, Kim MC, Han SU, Kim W, Ryu SW, Lee HJ, Song KY: Morbidity and mortality of laparoscopic gastrectomy versus open gastrectomy for gastric cancer: an interim report--a phase III multicenter, prospective, randomized Trial (KLASS Trial). Ann Surg 2010, 251:417-420.

32. Katai H, Sasako M, Fukuda H, Nakamura K, Hiki N, Saka M, Yamaue H, Yoshikawa T, Kojima K: Safety and feasibility of laparoscopy-assisted distal gastrectomy with suprapancreatic nodal dissection for clinical stage I gastric cancer: a multicenter phase II trial (JCOG 0703). Gastric Cancer 2010, 13:238-244.

33. Ouellette JR, Small DG, Termuhlen PM: Evaluation of Charlson-Age Comorbidity Index as predictor of morbidity and mortality in patients with colorectal carcinoma. J Gastrointest Surg 2004, 8:1061-1067.

34. Robbins JR, Gayar OH, Zaki M, Mahan M, Buekers T, Elshaikh MA: Impact of age-adjusted Charlson comorbidity score on outcomes for patients with early-stage endometrial cancer. Gynecol Oncol 2013, 131:593-597. 